\title{
Loss of DAB2IP Contributes to Cell Proliferation and Cisplatin Resistance in Gastric Cancer
}

This article was published in the following Dove Press journal:

OncoTargets and Therapy

\section{Guannan Wang* \\ Xu Wang* \\ Meng Han \\ Xiaoming Wang}

Department of Hepatobiliary Surgery, The First Affiliated Hospital of Wannan Medical College, Wuhu, 241000, People's Republic of China

*These authors contributed equally to this work
Correspondence: Xiaoming Wang

Department of Hepatobiliary Surgery,

The First Affiliated Hospital of Wannan

Medical College, No. 2 Zheshan West

Road, Jinghu District, Wuhu, 24I000,

Anhui Province, People's Republic of

China

Email wangxiaoming0203@sina.com
Objective: Resistance to chemotherapeutic drugs, such as cisplatin, has been one of the major problems adversely affecting the clinical prognosis of patients with gastric cancer (GC). Disabled Homolog 2-Interacting Protein (DAB2IP) status is one of the major factors involved in sensitivity to chemotherapy in multiple cancer types. In the present study, we aimed to investigate the potential roles of DAB2IP in GC cell proliferation and cisplatin resistance.

Materials and Methods: DAB2IP expression was detected in human GC tissues using immunohistochemistry (IHC). The role of DAB2IP in regulating GC cell proliferation and cisplatin resistance was explored by genetic manipulation. Western blot analysis was used to determine the molecular signaling to explain the mechanism of the observed DAB2IP effects in GC.

Results: DAB2IP expression was downregulated in human GC tissues and low DAB2IP expression predicted poor prognosis. Moreover, our data provided evidence that DAB2IP upregulation impaired cell proliferation property and sensitized GC cells to cisplatin while DAB2IP depletion possessed the opposite effects. Mechanistically, we showed that DAB2IP could inhibit the phosphorylation and activation of protein kinase B (AKT) and extracellular signal-regulated kinase (ERK), and the enhanced proliferation ability induced by DAB2IP knockdown was greatly impaired after incubation with AKT or ERK inhibitor.

Conclusion: DAB2IP modulates GC cell proliferation and sensitivity to cisplatin potentially via regulation of AKT and ERK signaling pathway, indicating that DAB2IP may serve as a potential prognostic biomarker and therapeutic target for treatment of GC.

Keywords: DAB2IP, proliferation, chemoresistance, gastric cancer

\section{Introduction}

Gastric cancer (GC) is one of the most common types of malignant cancer and the third leading cause of cancer-related deaths worldwide. ${ }^{1,2}$ Despite the improvement in diagnostic methods and perioperative management, GC patients in advanced stages frequently develop recurrence after radical resections, and consequently show poor survival rates. ${ }^{3,4}$ Surgery combined with chemotherapy is the primary treatment for GC. However, traditional chemotherapeutic drugs, such as cisplatin and fluorouracil, have limited efficacy in treating $\mathrm{GC}$ due to resistance to chemotherapy. ${ }^{5,6}$ Therefore, it is of great significance to identify molecules and mechanisms involved in drug resistance and to develop novel therapeutic targets and strategies for the improvement of chemotherapy against GC.

Disabled Homolog 2-Interacting Protein (DAB2IP), a RAS-GTPase-activating protein (RAS-GAP) family member, functions as a tumor suppressor and is 
downregulated in multiple aggressive cancers, ${ }^{7,8}$ such as pancreatic cancer, ${ }^{9}$ renal cancer, ${ }^{10}$ ovarian cancer, ${ }^{11}$ nasopharyngeal carcinoma, ${ }^{12}$ colorectal cancer, ${ }^{13}$ prostate cancer, ${ }^{14}$ and bladder cancer. ${ }^{15}$ In cancers, DAB2IP is known to regulate various biological process including cell survival, apoptosis, migration and invasion through affecting multiple oncogenic signaling pathways. ${ }^{711-16}$ Recent studies ${ }^{17-19}$ in prostate, bladder and renal cancer demonstrated that DAB2IP loss decreased cell sensitivity to chemotherapeutic agents or targeted drugs, suggesting a role for DAB2IP in chemoresistance in multiple cancer types. In consistency, previous studies ${ }^{20-22}$ also showed that DAB2IP was significantly downregulated in GC tissues and DAB2IP knockdown promoted GC cell proliferation, migration as well as activation of the protein kinase B (AKT) and extracellular signal-regulated kinase (ERK) signaling pathways. However, the prognostic value and potential role of DAB2IP in GC cell sensitivity to chemotherapy have not been elucidated.

In this study, we further explored the expression of DAB2IP in GC specimens and found that DAB2IP expression is downregulated in human GC tissues and low DAB2IP expression predicts poor prognosis. Moreover, our data provided evidence that DAB2IP upregulation impairs cell proliferation property and sensitizes GC cells to cisplatin while DAB2IP depletion has the opposite effects. Mechanistically, we showed that DAB2IP could inhibit the phosphorylation and activation of AKT and ERK, which may be implicated in DAB2IP regulation of GC cell proliferation and chemosensitivity. Taken together, these results suggest that DAB2IP may be a promising prognostic marker and a novel therapeutic target for the chemotherapy against GC.

\section{Materials and Methods Human GC Tissues}

Fifty-nine cases of primary gastric adenocarcinomas (GAC) and corresponding tumor-adjacent specimens were obtained from patients who underwent curative surgery from 2012 to 2013 at the Department of General Surgery, the First Affiliated Hospital of Wannan Medical College. All the clinicopathological information of patients is presented in Table 1. Informed consent from all patients was obtained. The Medical Ethics Committee of the First Affiliated Hospital of Wannan Medical College approved the present study according to the Declaration of Helsinki.

\section{Cell Lines and Cell Culture}

The human GC cell lines (AGS, MGC803, BGC823, SGC7901 and MKN45) and a normal gastric epithelium cell line (GES-1) were purchased from the Cell Bank of the Chinese Academy of Sciences (Shanghai, China). All the cells were cultured in Roswell Park Memorial Institute (RPMI; Hyclone) containing 10\% fetal bovine serum (FBS; Gibco) and 1\% penicillin/streptomycin (Gibco) at $37^{\circ} \mathrm{C}$ in a humidified atmosphere containing $5 \% \mathrm{CO}_{2}$. GC cell lines stably expressing DAB2IPspecific short hairpin RNA (shRNA) and plasmids encoding human DAB2IP were constructed to downregulate and upregulate DAB2IP respectively using a lentivirus technique (GeneChem, Shanghai, China). Lentiviral transduction was performed according to the manufacturer's instructions. The human DAB2IP shRNA target sequence is $5^{\prime}$-GGG AUA GGC UAA GGA GUAATT-3'.

\section{Immunohistochemistry (IHC)}

Briefly, the tissues that were previously formalin-fixed and paraffin-embedded were sliced into $4 \mu \mathrm{m}$ sections, and were then incubated with the primary antibody recognizing human DAB2IP (\#ab87811, abcam) at 1:200 dilution at $4{ }^{\circ} \mathrm{C}$ overnight. The protein was visualized using a tissue staining kit (Zhongshan Biotechnology, Beijing, China) according to the manufacturer's instructions. The final staining score was determined by color intensity and positive cell rate, ranging $0-12$ which was described in our previous study. ${ }^{23,24}$ Patients were classified into two groups: scores $0-4$ were considered as none or low, while 5-12 were considered as high expression.

\section{Protein Extraction and Western Blot Analysis}

Cells were lysed in RIPA lysis buffer (Beyotime Institute of Biotechnology) supplemented with cocktails of protease and phosphatase inhibitors (Sigma). A total of $20 \mu \mathrm{g}$ protein was then separated by $8 \%$ SDS-PAGE and then transferred onto a PVDF membranes according to methods described previously. ${ }^{23,24}$ Antibodies against DAB2IP (dilution 1:1000; \#ab87811, abcam), cyclin D1 (dilution 1:1000; 26939-1-AP, proteintech), p21 (dilution 1:1000; 10355-1-AP, proteintech), p-AKT (Ser473) (dilution 1:1000; \#4058, CST), AKT (dilution 1:1000; \#9272, CST), p-ERK (dilution 1:1000; \#4370, CST), ERK 
Table I Relationships Between DAB2IP Expression and Clinic-Pathological Factors in 59 GC Patients

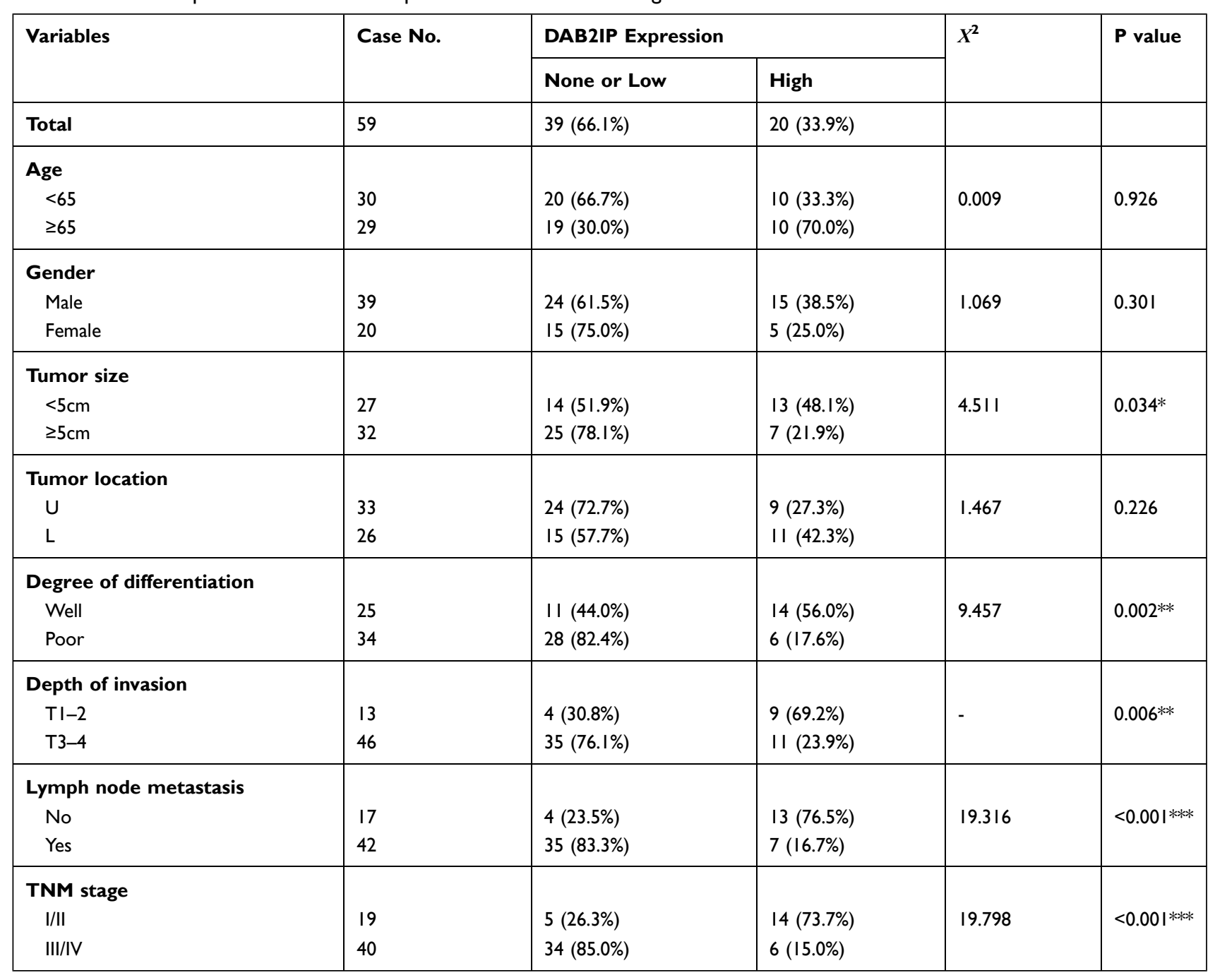

Notes: $* \mathrm{P}<0.05 ; * * \mathrm{P}<0.01 ; * * * \mathrm{P}<0.001$.

(dilution 1:1000; \#4695, CST) and Tubulin (dilution 1:2000; \#AF5012, Beyotime) were used in this study.

\section{RNA Extraction and Quantitative Real-Time PCR (q-PCR)}

Total RNA was exacted using TRIzol reagent (Thermo Fisher Scientific) following the manufacturer's instruction. q-PCR was performed on ABI 7500 Fast Real-Time PCR (RT-PCR) System (Applied Biosystems, Waltham, MA, UK) with SYBR Green RT-PCR kits (TaKaRa). The expression of DAB2IP was normalized to that of human $18 \mathrm{~s}$. The primer sequences used in the study were: humanDAB2IP-Forward, 5'-CTG AGC GGG ATA AGT GGA TGG-3', human-DAB2IP-Reverse, 5'-AAA CAT TGT CCG TCT TGA GCTT-3', human-18s-Forward, 5'-GTA
ACC CGT TGA ACC CCATT-3', human-18s-Reverse, 5'CCA TCC AAT CGG TAG TAGCG-3'.

\section{Cell Viability Assay}

Cell proliferation and the half maximal inhibitory concentration (IC50) of each cell line to cisplatin were evaluated using an MTT assay kit (Amresco, USA). Cells were seeded into 96-well plates for 24 hours and treated with or without cisplatin at different concentrations for 48 hours. Then it was incubated with MTT solution-containing culture medium at $37^{\circ} \mathrm{C}$ for 4 hours and supernatants were then removed. Formazan crystals were dissolved in $150 \mu \mathrm{L}$ dimethyl sulfoxide (DMSO) with gentle shaking for 10 minutes, and the absorbance value (OD) was measured at $490 \mathrm{~nm}$. 


\section{Colony Formation Assay}

Cells were seeded in 6-well plates for $24 \mathrm{~h}$, and then switched into fresh medium containing $4 \mu \mathrm{M}$ cisplatin, $20 \mu \mathrm{M}$ Ly294002 or $20 \mu \mathrm{M}$ U0126 for two weeks, and fresh medium was changed every 3 days. The plates were then fixed and stained with $0.1 \%$ crystal violet and the images were taken by a digital camera (Nikon).

\section{Statistical Analysis}

Data are presented as mean \pm standard error of the mean (SEM). Statistical significance was analyzed using a Student's $t$-test (paired or unpaired, two-tailed) or chisquare test. Survival duration was calculated using the KaplanMeier method and compared using the Log rank test. A value of $P<0.05$ was considered as statistically significant.

\section{Results}

\section{DAB2IP Expression is Downregulated in} Human GC Tissues and Low DAB2IP Expression Predicts Poor Prognosis

Given that DAB2IP functions as a tumor suppressor in many cancers, ${ }^{7,8}$ we further investigated the expression of DAB2IP in GC tumor tissues and corresponding tumoradjacent normal tissues using IHC staining. Only 33.90\% (20/59) samples of GC tissue exhibited high staining of DAB2IP, while high DAB2IP signal were detected in $86.44 \%(51 / 59)$ of tumor-adjacent specimens $(P<0.001$, Figure 1A and B). Further analysis revealed that DAB2IP expression in GC patients with lymph node metastasis (LNM) was further reduced compared with those without LNM $(P<0.001$, Figure $1 C)$. In addition, GC tumor tissues with deeper invasion (T3-4), larger tumor size $(\geq$ $5 \mathrm{~cm}$ ) and advanced tumor-node-metastasis (TNM) stage (III-IV) showed lower DAB2IP expression than those with T1-2 $(P<0.01$, Figure 1D), tumor size $<5 \mathrm{~cm}(P<$ 0.05 , Figure 1E) and TNM stage I-II $(P<0.001$, Figure $1 \mathrm{~F}$ ), respectively. Besides, cytologic experiments (Figure $1 \mathrm{G})$ showed that DAB2IP expression in a normal gastric epithelium cell line (GES-1) was higher than most of GC cell lines (AGS, MGC803, BGC823, SGC7901 and MKN45) detected in this study.

Next, we evaluated the correlations between DAB2IP expression and clinicopathologic features of GC patients. As shown in Table 1, DAB2IP expression was significantly correlated with tumor size $(P=0.034)$, degree of differentiation $(P=0.002)$, depth of invasion $(P=0.006)$, LNM $(P<0.001)$ and TNM stage $(P<0.001)$. However, there was no significant differences regarding age, gender or tumor location $(P>0.05$, Table 1). Moreover, Kaplan-Meier curves indicated that low DAB2IP expression was associated with poor outcomes in patients suffering GC (Log rank test, $P=0.005$, Figure $1 \mathrm{H}$ ), which suggested the prognostic value of DAB2IP in GC.

\section{Stable Knockdown and Overexpression of DAB2IP in GC Cells}

As shown in Figure 1G, there was relatively high DAB2IP protein expression in AGS, SGC7901 and MGC803 cell lines, but low expression of DAB2IP in BGC823 and MKN45 cell lines. To explore the molecular mechanism of DAB2IP involved in the progression of GC, we knocked down DAB2IP in AGS and SGC7901 cells with high endogenous DAB2IP level and generated stable transfectants. Results from Western blots and q-PCR showed that DAB2IP expression at both protein and mRNA levels were significantly decreased in AGS and SGC7901 cells stably transfected with DAB2IP shRNA (KD) compared with those transfected with control shRNA (NC, $P<0.001$, Figure 2A). Similarly, we introduced plasmids encoding human DAB2IP or empty vector into BGC823 and MKN45 cells which express low endogenous DAB2IP. The overexpression efficiency was determined by Western blotting and q-PCR, and results showed that both DAB2IP protein and mRNA expression levels were higher in cells transfected with plasmids encoding human DAB2IP (OE) than in cells transfected with empty vector (VEC, $P<0.001$, Figure $2 \mathrm{~B}$ ).

\section{DAB2IP Inhibits GC Cell Proliferation in vitro}

In view of the above integrated analysis results, we asked whether DAB2IP is involved in regulating GC cell phenotypes. First, MTT assays were used to analyze the effect of DAB2IP on cell proliferation property of GC. We found that DAB2IP silencing greatly promoted AGS cell proliferation compared to the control cells (Figure 3A). Conversely, BGC823 cells with stable DAB2IP overexpression showed lower proliferation ability than cells transfected with empty vector (Figure 3B). In line with the results of MTT assays, colony formation assays showed that AGS cells with stable DAB2IP knockdown presented a superior ability to form foci $(P<0.01$, Figure $3 C)$, whereas the ability of BGC 823 cells to form foci was greatly impaired when cells overexpressed DAB2IP $(P<0.01$, Figure 3D). In addition, we examined the effects of DAB2IP on G1-S transition 
A

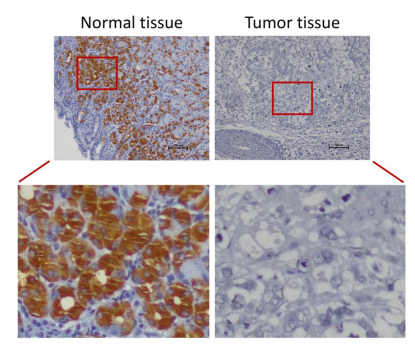

D

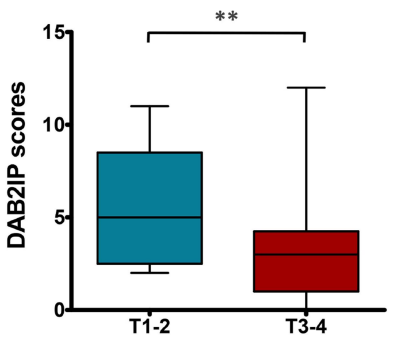

B

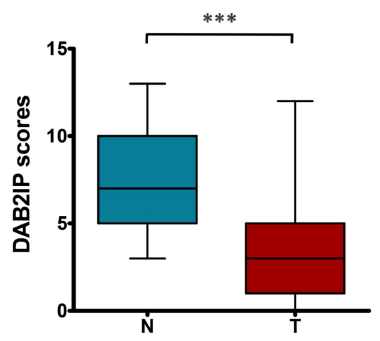

E

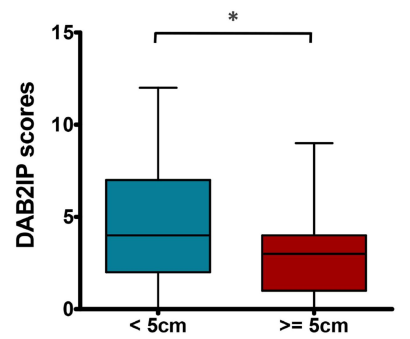

C

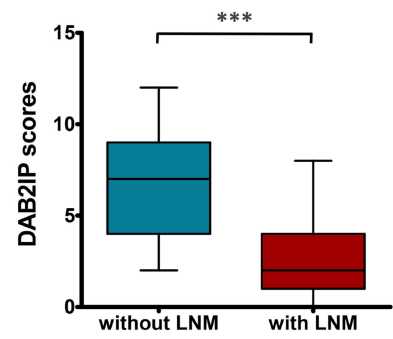

$\mathbf{F}$

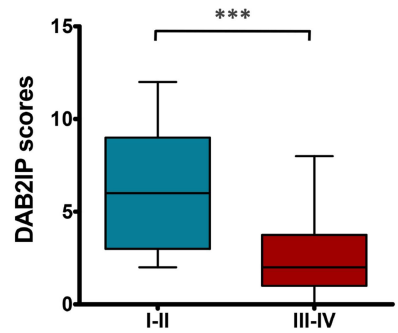

H
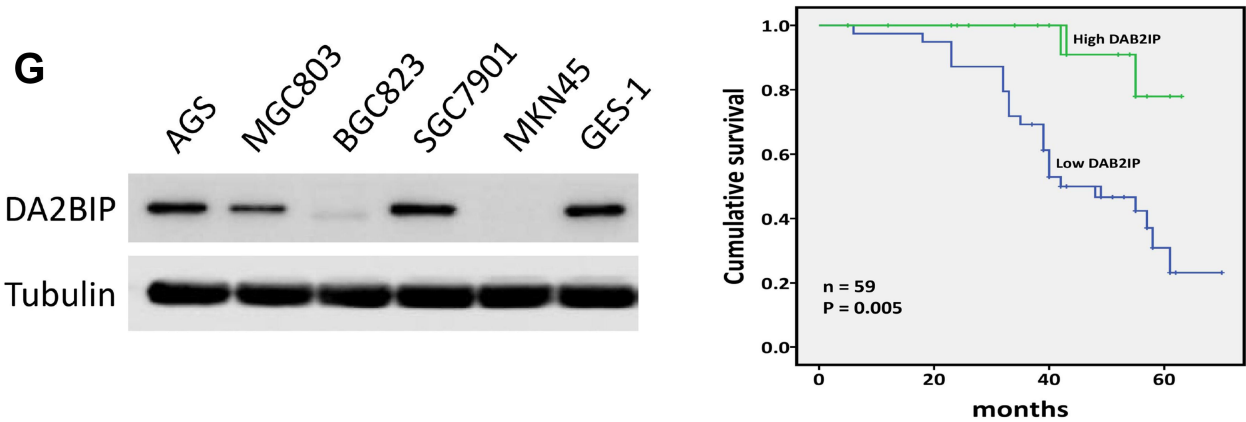

Figure I DAB2IP expression in human GC tissues and cell lines. (A) IHC staining of DAB2IP in normal tissue and in GC tumor tissue. (B-F) IHC scores of DAB2IP in (B) normal tissues (N) vs tumor tissues (T), (C) tumors with or without LNM, (D) depth of invasion (TI-2 vs T3-4), (E) tumor size (<5cm vs $\geq 5 \mathrm{~cm})$, and $(\mathbf{F})$ TNM stage (I-II vs III-IV). (G) Western blot analysis of DAB2IP expression in five GC (AGS, MGC803, BGC823, SGC790I and MKN45) and non-GC GES-I cell lines. Tubulin as a loading control. $(\mathbf{H})$ Kaplan-Meier analysis (Log rank test) of the overall survival of GC patients with high or low DAB2IP expression levels. $* P<0.05$, $* * P<0.0 \mathrm{I}$, $* * * P<0.00 \mathrm{I}$.

promoter cyclin D1 and G1 gatekeepers p21 in GC cells. Our results showed that DAB2IP knockdown decreased the expression level of p21 and increased the expression of cyclin D1, while ectopic expression of DAB2IP did the opposite (Figure $3 \mathrm{E}-\mathrm{G}$ ), which confirmed the inhibitory effect of DAB2IP in GC cell proliferation.

\section{DAB2IP Modulates the Chemosensitivity of GC Cells to Cisplatin}

To determine the potential role of DAB2IP in regulating chemosensitivity of GC cells to cisplatin, MTT assays were performed in AGS (NC vs KD) and BGC823 (VEC vs $\mathrm{OE})$ cells. In general, AGS cells with stable DAB2IP knockdown showed significantly higher resistance to cisplatin, with increases in IC50 value compared to the control group (Figure 4A). On the other hand, BGC823 cells with stable DAB2IP overexpression became more sensitive to cisplatin compared with the negative control (Figure 4B). In consistency, we found that knockdown of DAB2IP could dramatically enhance the colony formation ability of AGS cells with cisplatin treatment (Figure 4C). Furthermore, ectopic expression of DAB2IP in MKN45 cells could greatly enhance cell sensitivity to cisplatin in a dose-dependent manner (Figure 4D and E). Together, these data indicated that loss of DAB2IP attenuates cellular sensitivity to cisplatin. 

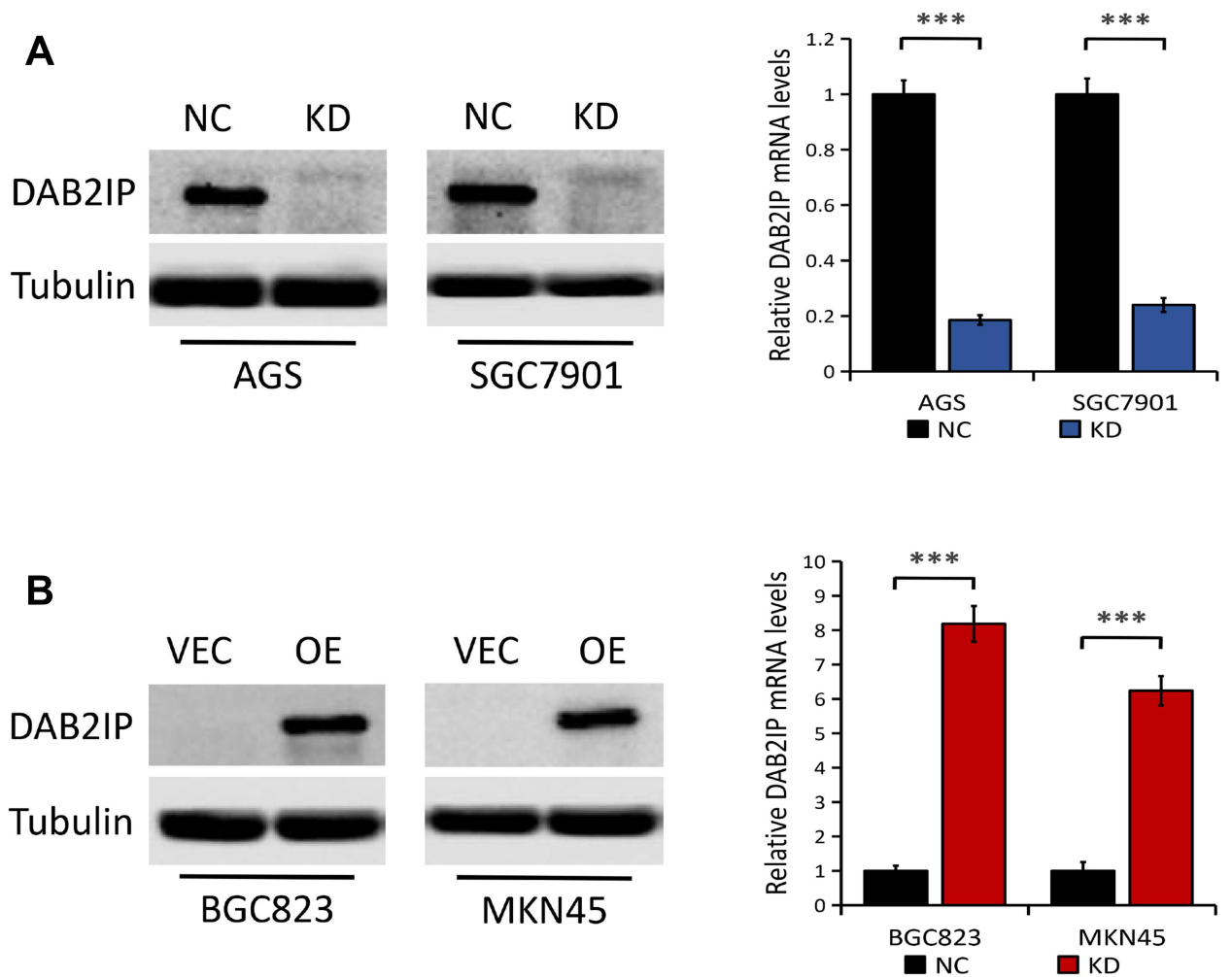

Figure 2 Knockdown and overexpression efficiency of DAB2IP in GC cells. (A) The protein (left) and mRNA (right) expression of DAB2IP in AGS and SGC790I cells stably transfected with either control shRNA (NC) or DAB2IP shRNA (KD). (B) The protein (left) and mRNA (right) expression of DAB2IP in BGC823 and MKN45 cells stably transfected with either empty vector (VEC) or plasmids encoding human DAB2IP (OE). Tubulin or I8s as the loading control. $* * * P<0.00 \mathrm{I}$.

\section{DAB2IP Regulates AKT and ERK Signaling Pathway in GC Cells}

To understand the underlying mechanisms of DAB2IP in this event, we explored possible downstream signaling pathways such as AKT and ERK, which are both closely associated with cancer cell proliferation and chemoresistance. ${ }^{25-27}$ In line with previous studies, ${ }^{20-22}$ we further confirmed the inhibitory effect of DAB2IP on AKT and ERK signaling pathway. We found that DAB2IP depletion notably increased the levels of phosphorylated AKT (p-AKT) and phosphorylated ERK (p-ERK) in both AGS and SGC7901 cells, but not their total protein levels (Figure 5A). On the contrary, ectopic expression of DAB2IP had the opposite effect on the phosphorylation levels of AKT and ERK (Figure 5B).

To further explore whether AKT and ERK signaling pathway are implicated in DAB2IP suppression of GC cell proliferation, we treated DAB2IP-deficient cells with Ly294002 (an indirect inhibitor of AKT) and U0126 (a highly selective inhibitor of ERK). Colony formation assays showed that the enhanced proliferation ability induced by DAB2IP knockdown was greatly impaired after incubation with Ly294002 or U0126 in SGC7901 cells (Figure 5C). Moreover, combined inhibition of AKT and ERK signaling pathway in DAB2IP-depleted SGC7901 cells weakened cell proliferation ability further.

\section{Discussion}

Increasing evidence has demonstrated that DAB2IP functions as a tumor suppressor and participates in cell survival, apoptosis, migration and invasion in multiple types of cancer. ${ }^{7,11-16}$ Loss of DAB2IP also confers resistance to chemotherapy in prostate, bladder and renal cancers, ${ }^{17-19}$ suggesting a role for DAB2IP as a potential target for cancer therapy. In GC, ${ }^{20-22}$ DAB2IP was reported to be downregulated and DAB2IP knockdown enhanced the ability of cell proliferation and migration, but the potential effect of DAB2IP on chemosensitivity and the internal mechanism remain unclear and need to be explored.

In the present study, we provided further evidence that DAB2IP expression was downregulated in human GC tissues compared to corresponding tumor-adjacent normal tissues. Further analysis showed that DAB2IP expression in GC patients with LNM, deeper invasion (T3-4), larger tumor size $(\geq 5 \mathrm{~cm})$ and advanced TNM stage (III-IV) was further reduced compared with those without LNM, T1-2, 
A

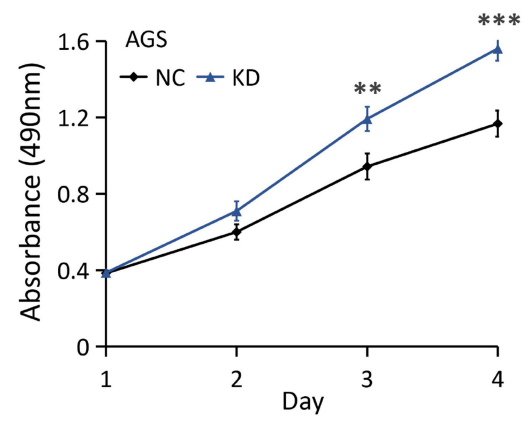

C

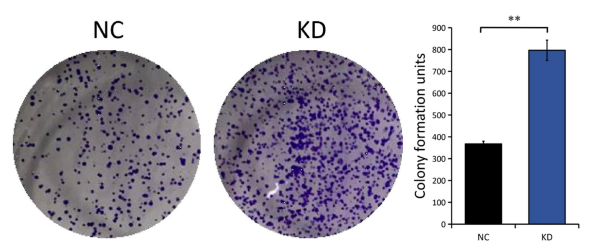

B

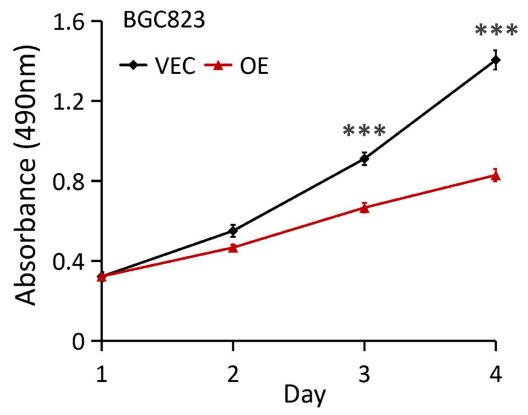

D
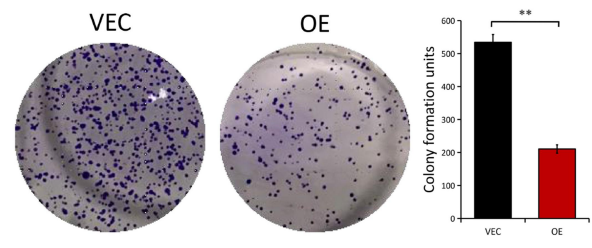

E

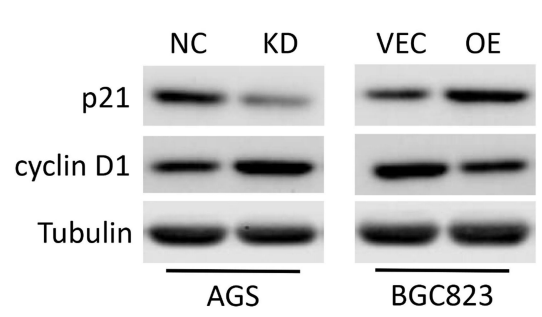

F

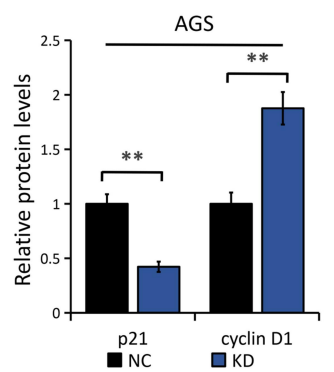

G

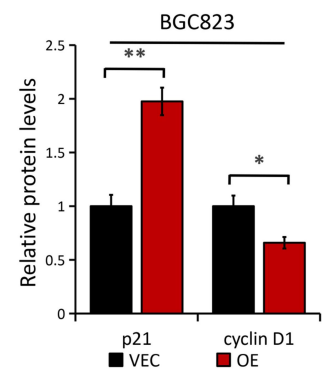

Figure 3 Effect of DAB2IP expression on GC cell proliferation. (A) MTT assay of AGS cells stably transfected with either control shRNA (NC) or DAB2IP shRNA (KD). (B) MTT assay of BGC823 cells stably transfected with either empty vector (VEC) or plasmids encoding human DAB2IP (OE). (C) Colony formation assay of AGS cells (NC vs KD). The bands were quantified and presented as the mean \pm SEM. (D) Colony formation assay of BGC823 cells (VEC vs OE). The bands were quantified and presented as the mean \pm SEM. (E-G) Western blot analysis (E) of the indicated proteins in AGS (NC vs KD) and BGC823 (VEC vs OE) cells. Tubulin as a loading control. The bands ( $\mathbf{F}$ and $\mathbf{G})$ were quantified and presented as the mean \pm SEM. $* * P<0.01$, *** $p<0.001$.

tumor size $<5 \mathrm{~cm}$ and TNM stage $\mathrm{I}-\mathrm{II}$, respectively. Clinical data analyses revealed that low DAB2IP expression in GC tissues was significantly associated with tumor size, degree of differentiation, depth of invasion, LNM, TNM stage and poor prognosis. Moreover, we also evidenced that DAB2IP upregulation decreased cell proliferation and sensitized GC cells to cisplatin while DAB2IP depletion did the opposite.

DAB2IP, acting as a signaling adaptor, modulates multiple key oncogenic pathways, including $\mathrm{TNF} \alpha / \mathrm{NF}-\kappa \mathrm{B}$, WNT/ $\beta$-catenin, JAK/STAT, PI3K/AKT and RAS/ERK. ${ }^{7,8}$ Enhanced cell proliferation was reported to be correlated to abrogation of DAB2IP functions in various cultured cell lines, and has been linked to hyperactivation of AKT and ERK. ${ }^{7,16,21}$ Resistance to chemotherapy is a significant barrier to cure cancer and multiple molecular mechanisms are involved in chemoresistance. In prostate cancer, ${ }^{17}$ DAB2IP loss was shown to enhance chemoresistance through upregulation of the antiapoptotic protein Clusterin. In bladder cancer, ${ }^{18}$ DAB2IP was reported to modulate chemoresistance to pirarubicin by regulation of STAT3/Twist1/ P-glycoprotein signaling. In the present study, we further explored the the underlying mechanisms of DAB2IP regulation of GC cell proliferation and chemosensitivity. AKT and ERK, as major downstream signaling pathways of DAB2IP, are crucial for cancer cell proliferation and chemoresistance. $^{25-27}$ In accordance with the previous studies, ${ }^{20-22}$ we further confirmed the inhibitory effect of DAB2IP on AKT and ERK signaling pathway in GC cells and the enhanced proliferation ability induced by DAB2IP knockdown was greatly impaired after incubation with AKT or ERK inhibitor. Moreover, combined inhibition of AKT 
A
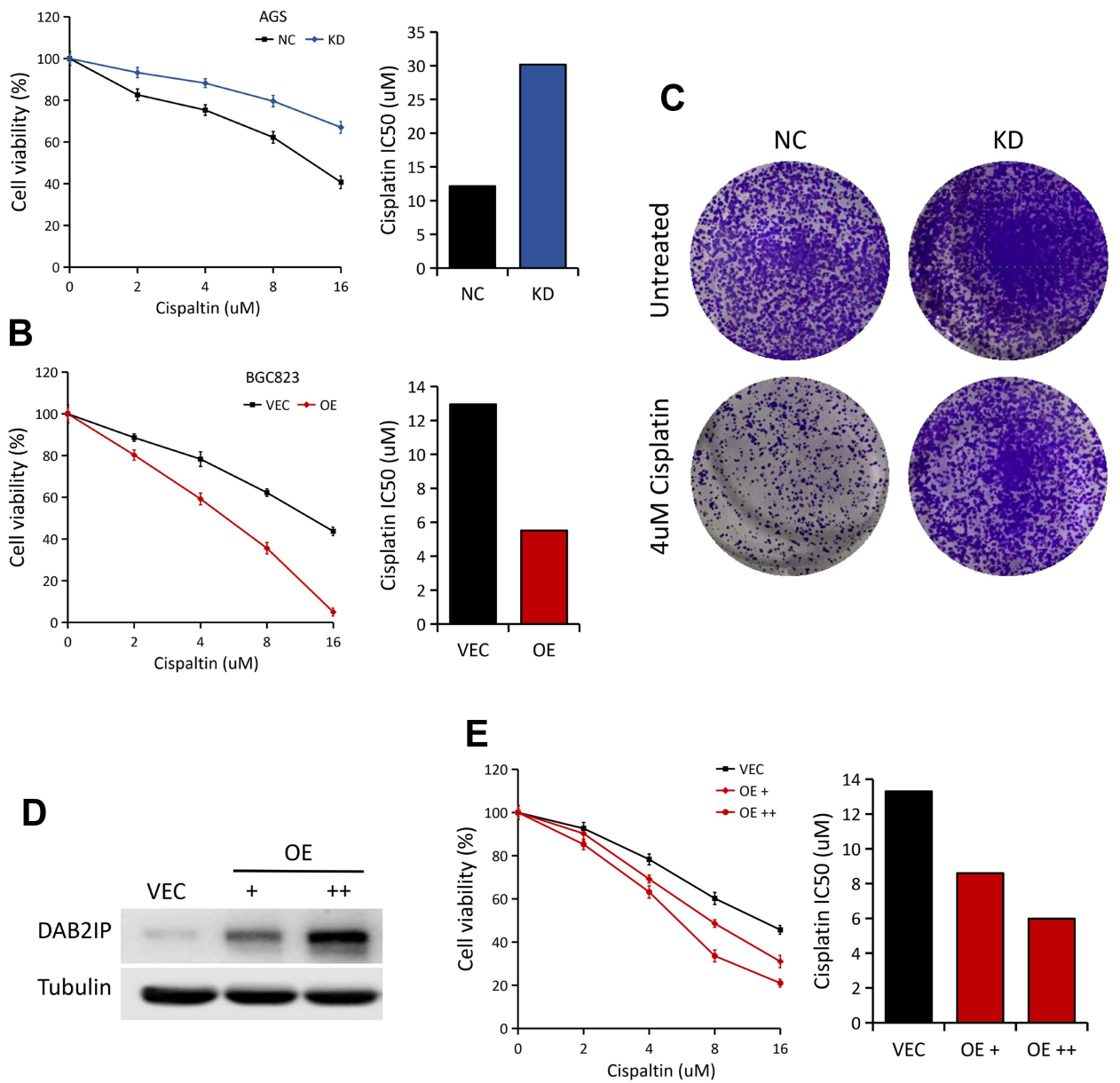

Figure 4 Loss of DAB2IP in GC cells promotes resistance to cisplatin. (A) Cell viabilities of AGS cells (NC vs KD) treated with different concentrations of cisplatin for 48 h. IC50 of cisplatin for AGS was presented (right). (B) Cell viabilities of BGC823 cells (VEC vs OE) treated with different concentrations of cisplatin for 48 h. IC50 of cisplatin for BGC823 was presented (right). (C) AGS cells (NC vs KD) with $4 \mu \mathrm{M}$ cisplatin treatment were subjected to colony formation assay, the representative pictures were shown. (D) Western blot analysis of DAB2IP protein in MKN45 cells with increasing amounts of DAB2IP. Tubulin as a loading control. (E) Cell viabilities of MKN45 cells (VEC vs OE) treated with different concentrations of cisplatin for $48 \mathrm{~h}$. IC50 of cisplatin was presented (right).

and ERK signaling pathway in DAB2IP-depleted SGC7901 cells weakened cell proliferation ability further. These data indicated that DAB2IP modulates GC cell proliferation and sensitivity to cisplatin potentially via regulation of AKT and ERK signaling pathway.

DAB2IP has a relatively long 3'UTR sequence, and is therefore a good candidate for post-transcriptional silencing by microRNAs (miRNAs). ${ }^{7}$ miR-889 was reported to downregulate DAB2IP protein level and DAB2IP overexpression can subvert miR-889-induced proliferation phenotype in esophageal squamous cell carcinoma (ESCC) cells. ${ }^{28}$ In prostate cancer, the oncogenic miR-32 was also shown to target DAB2IP. ${ }^{29}$ Moreover, miR-92b was reported to promote GC cell proliferation by activating the DAB2IP-mediated PI3K/AKT signalling pathway. $^{22}$ Together, these studies confirm that DAB2IP is a target of miRNA-mediated repression, and suggest miRNA/DAB2IP signalling axis may be a potential therapeutic target in cancer treatment.

There are some limitations in our study. Firstly, in vivo study should be conducted to determine the effects of DAB2IP on GC cell growth and sensitivity to cisplatin. Secondly, though AKT and ERK signaling pathway may be implicated in DAB2IP regulation of GC cell proliferation and cisplatin resistance, the precise molecular mechanism still warrants further investigation.

In conclusion, this study not only highlights DAB2IP as a potential marker in prognosticating the outcome of patients with $\mathrm{GC}$, but also emphasizes its role in regulating GC cell proliferation and sensitivity to cisplatin. Mechanistically, hyperactivation of AKT and ERK may be implicated in DAB2IP depletion-mediated increase of 

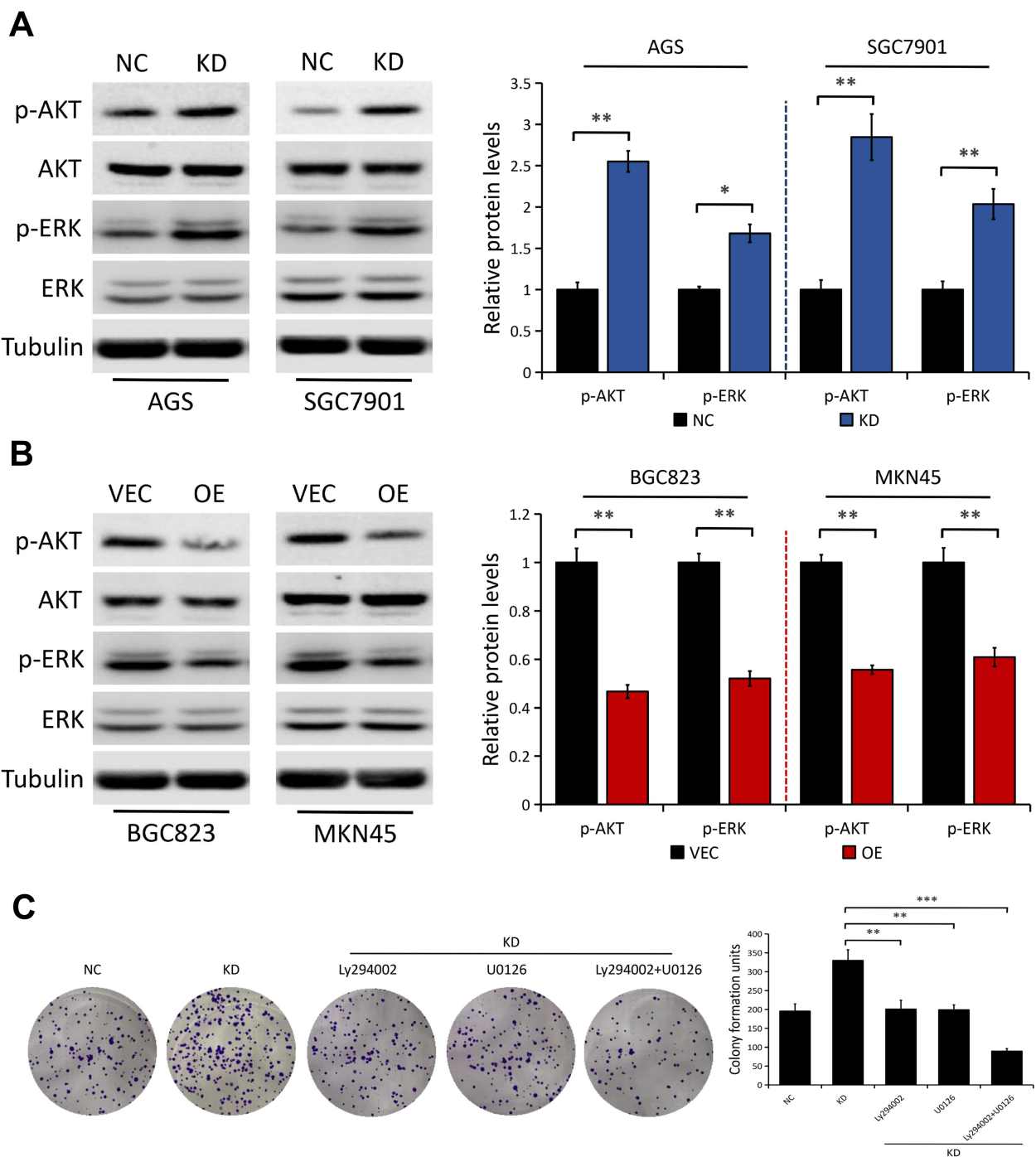

Figure 5 DAB2IP regulates the phosphorylation of AKT and ERK in GC cells. (A) Western blot analysis of the indicated proteins in AGS and SGC790I cells stably transfected with either control shRNA (NC) or DAB2IP shRNA (KD). Tubulin as a loading control. The bands were quantified and presented as the mean \pm SEM. (B) Western blot analysis of the indicated proteins in BGC823 and MKN45 cells stably transfected with either empty vector (VEC) or plasmids encoding human DAB2IP (OE). Tubulin as a loading control. The bands were quantified and presented as the mean \pm SEM. (C) Colony formation assay of SGC790I cells (NC vs KD) with or without treatment of Ly294002 $(20 \mu \mathrm{M})$ or U0I26 $(20 \mu \mathrm{M})$. The bands were quantified and presented as the mean \pm SEM. $* P<0.05$, **P $<0.01$, $* * * P<0.001$.

cell proliferation and cisplatin resistance, indicating DAB2IP as a potential target for developing more effective therapy against $\mathrm{GC}$ in future.

\section{Funding}

This study was supported by the Anhui Provincial Central Government's Special Program for Local Science and Technology Development (Grant No. YDZX20183400004899), University Academic (Professional) Top Talents Academic Funding Project (Grant No. gxbjZD17), grants from the First Affiliated Hospital of Wannan Medical College (Grant No.
KGF2019703 and KPF2019011), and the Nature and Science Fund from Wannan Medical College, CN (Grant No. WK2019F07).

\section{Disclosure}

All authors declare that they have no conflicts of interest.

\section{References}

1. Ferlay J, Colombet M, Soerjomataram I, et al. Global cancer statistics 2018: GLOBOCAN estimates of incidence and mortality worldwide for 36 cancers in 185 countries. CA Cancer J Clin. 2018;68:394-424. doi:10.3322/caac. 21492 
2. Van Cutsem E, Sagaert X, Topal B, et al. Gastric cancer. Lancet. 2016;388:2654-2664. doi:10.1016/S0140-6736(16)30354-3

3. Ajani JA, Lee J, Sano T, et al. Gastric adenocarcinoma. Nat Rev Dis Primers. 2017;3:17036. doi:10.1038/nrdp.2017.36

4. Chen W, Zheng R, Baade PD, et al. Cancer statistics in China, 2015. CA Cancer J Clin. 2016;66(2):115-132. doi:10.3322/caac.21338

5. Kamiyama K, Sawada S, Fukuda T, et al. A case of long-term survival following two-stage surgery for perforated advanced gastric cancer caused by chemotherapy. Gan to Kagaku Ryoho. 2019;46:701-704

6. Ghosn M, Tabchi S, Kourie HR, et al. Metastatic gastric cancer treatment: second line and beyond. World J Gastroenterol. 2016;22 (11):3069-3077. doi:10.3748/wjg.v22.i11.3069

7. Bellazzo A, Di Minin G, Collavin L, et al. Block one, unleash a hundred. Mechanisms of DAB2IP inactivation in cancer. Cell Death Differ. 2017;24:15-25. doi:10.1038/cdd.2016.134

8. Liu L, Xu C, Hsieh J-T, et al. DAB2IP in cancer. Oncotarget. 2016;7:3766-3776. doi:10.18632/oncotarget.6501

9. Duan Y, Yin X, Lai X, et al. Upregulation of DAB2IP inhibits Ras activity and tumorigenesis in human pancreatic cancer cells. Technol Cancer Res Treat. 2020;19:1-11. doi:10.1177/1533033819895494

10. Yun EJ, Lin CJ, Dang A, et al. Downregulation of human DAB2IP gene expression in renal cell carcinoma results in resistance to ionizing radiation. Clin Cancer Res. 2019;25(14):4542-4551. doi:10.1158/ 1078-0432.CCR-18-3004

11. Fan X, Wang Y, Fan J, et al. Deletion of SMURF 1 represses ovarian cancer invasion and EMT by modulating the DAB2IP/AKT/Skp2 feedback loop. $J$ Cell Biochem. 2019;120(6):10643-10651. doi: $10.1002 /$ jcb. 28354

12. Wang B, Gu Q, Li J. DOC-2/DAB2 interactive protein regulates proliferation and mobility of nasopharyngeal carcinoma cells by targeting PI3K/Akt pathway. Oncol Rep. 2017;38(1):317-324. doi:10.3892/or.2017.5704

13. Zhu XH, Wang JM, Yang SS, et al. Down-regulation of DAB2IP promotes colorectal cancer invasion and metastasis by translocating hnRNPK into nucleus to enhance the transcription of MMP2. Int J Cancer. 2017;141(1):172-183. doi:10.1002/ijc.30701

14. Wang B, Huang J, Zhou J, et al. DAB2IP regulates EMT and metastasis of prostate cancer through targeting PROX1 transcription and destabilizing HIF1 $\alpha$ protein. Cell Signal. 2016;8(11):1623-1630. doi:10.1016/j.cellsig.2016.07.011

15. Shen YJ, Kong ZL, Wan FN, et al. Downregulation of DAB2IP results in cell proliferation and invasion and contributes to unfavorable outcomes in bladder cancer. Cancer Sci. 2014;105(6):704-712. doi: $10.1111 /$ cas. 12407

16. Xie D, Gore C, Zhou J, et al. DAB2IP coordinates both PI3K-Akt and ASK1 pathways for cell survival and apoptosis. Proc Natl Acad Sci U S A. 2009;106(47):19878-19883. doi:10.1073/pnas.09084 58106
17. Wu K, Xie D, Zou Y, et al. The mechanism of DAB2IP in chemoresistance of prostate cancer cells. Clin Cancer Res. 2013;19 (17):4740-4749. doi:10.1158/1078-0432.CCR-13-0954

18. Wu K, Wang B, Chen Y, et al. DAB2IP regulates the chemoresistance to pirarubicin and tumor recurrence of non-muscle invasive bladder cancer through STAT3/Twist1/P-glycoprotein signaling. Cell Signal. 2015;27(12):2515-2523. doi:10.1016/j.cellsig.2015.09.014

19. Zhou J, Luo J, Wu K, et al. Loss of DAB2IP in RCC cells enhances their growth and resistance to mTOR-targeted therapies. Oncogene. 2016;35(35):4663-4674. doi:10.1038/onc.2016.4

20. Tao Y, Sun C, Zhang T, et al. SMURF1 promotes the proliferation, migration and invasion of gastric cancer cells. Oncol Rep. 2017;38 (3):1806-1814. doi:10.3892/or.2017.5825

21. Sun L, Yao Y, Lu T, et al. DAB2IP downregulation enhances the proliferation and metastasis of human gastric cancer cells by derepressing the ERK1/2 pathway. Gastroenterol Res Pract. 2018;2018:2968252. doi:10.1155/2018/2968252

22. Ni QF, Zhang Y, Yu JW, et al. miR-92b promotes gastric cancer growth by activating the DAB2IP-mediated PI3K/AKT signalling pathway. Cell Prolif. 2020;53(1):e12630. doi:10.1111/cpr.12630

23. Cheng Z, Shao X, Xu M, et al. Rab1A promotes proliferation and migration abilities via regulation of the HER2/AKT-independent mTOR/S6K1 pathway in colorectal cancer. Oncol Rep. 2019;41 (5):2717-2728. doi:10.3892/or.2019.7071

24. Xu M, Shao X, Kuai X, et al. Expression analysis and implication of Rab1A in gastrointestinal relevant tumor. Sci Rep. 2019;9(1):13384. doi:10.1038/s41598-019-49786-7

25. Li W, Wang L, Ji XB, et al. MiR-199a inhibits tumor growth and attenuates chemoresistance by targeting K-RAS via AKT and ERK signalings. Front Oncol. 2019;9:1071. doi:10.3389/fonc.2019.01071

26. Wang T, Chen G, Ma X, et al. MiR-30a regulates cancer cell response to chemotherapy through SNAI1/IRS1/AKT pathway. Cell Death Dis. 2019;10(3):153. doi:10.1038/s41419-019-1326-6

27. Pan CW, Jin X, Zhao Y, et al. AKT-phosphorylated FOXO1 suppresses ERK activation and chemoresistance by disrupting IQGAP1-MAPK interaction. EMBO J. 2017;36(8):995-1010. doi: $10.15252 / \mathrm{embj} .201695534$

28. $\mathrm{Xu} \mathrm{Y,} \mathrm{He} \mathrm{J,} \mathrm{Wang} \mathrm{Y,} \mathrm{et} \mathrm{al.} \mathrm{miR-889} \mathrm{promotes} \mathrm{proliferation} \mathrm{of}$ esophageal squamous cell carcinomas through DAB2IP. FEBS Lett. 2015;589:1127-1135. doi:10.1016/j.febslet.2015.03.027

29. Liao H, Xiao Y, Hu Y, et al. MicroRNA-32 induces radioresistance by targeting DAB2IP and regulating autophagy in prostate cancer cells. Oncol Lett. 2015;10:2055-2062. doi:10.3892/ol.2015.3551

\section{Publish your work in this journal}

OncoTargets and Therapy is an international, peer-reviewed, open access journal focusing on the pathological basis of all cancers, potential targets for therapy and treatment protocols employed to improve the management of cancer patients. The journal also focuses on the impact of management programs and new therapeutic agents and protocols on patient perspectives such as quality of life, adherence and satisfaction. The manuscript management system is completely online and includes a very quick and fair peer-review system, which is all easy to use. Visit http://www.dovepress.com/ testimonials.php to read real quotes from published authors. 